\title{
Circularly polarized luminescent systems fabricated by Tröger's base derivatives through two different strategies
}

\author{
Cheng Qian ${ }^{\ddagger 1}$, Yuan Chen ${ }^{\ddagger 1}$, Qian Zhao ${ }^{1}$, Ming Cheng ${ }^{1}$, Chen Lin ${ }^{1}$, Juli Jiang ${ }^{* 1}$ \\ and Leyong Wang ${ }^{*}{ }^{1,2}$
}

Open Access

\author{
Letter \\ Address: \\ ${ }^{1}$ Key Laboratory of Mesoscopic Chemistry of MOE, Jiangsu Key \\ Laboratory of Advanced Organic Materials, School of Chemistry and \\ Chemical Engineering, Nanjing University, Nanjing, 210023, China \\ and ${ }^{2}$ Advanced Materials Institute, Qilu University of Technology \\ (Shandong Academy of Sciences), Jinan, 250014, China \\ Email: \\ Juli Jiang* - jjl@nju.edu.cn; Leyong Wang* - lywang@nju.edu.cn \\ * Corresponding author $\ddagger$ Equal contributors \\ Keywords: \\ circularly polarized luminescence; chiral resolution; co-gelation; \\ inversion of CPL handedness; Tröger's base
}

Beilstein J. Org. Chem. 2021, 17, 52-57. https://doi.org/10.3762/bjoc.17.6

Received: 01 October 2020

Accepted: 09 December 2020

Published: 06 January 2021

This article is part of the thematic issue "Molecular recognition" and is dedicated to the memory of Carsten Schmuck.

Guest Editor: J. Niemeyer

(C) 2021 Qian et al.; licensee Beilstein-Institut. License and terms: see end of document.

\begin{abstract}
The Tröger's base derivative rac-TBPP was synthesized and separated into two enantiomers $R_{2 N}$-TBPP and $S_{2 N}$-TBPP by chiral column chromatography. These compounds show a strong circularly polarized luminescence with $g_{\text {lum }}$ values of +0.0021 , and -0.0025 , respectively. The second way to fabricate the rac-TBPP-based CPL-active material is to co-gel the fluorescent rac-TBPP with a chiral D-glutamic acid gelator DGG by co-assembly strategy. At the molar ratio of $r a c$-TBPP/DGG $=1: 80$, the $g_{\text {lum }}$ value of the co-gel was about three times higher than the $g_{\text {lum }}$ values of $R_{2 N^{-}}$TBPP and $S_{2 N}$-TBPP enantiomers. Interestingly, the CPL handedness of the rac-TBPP/DGG co-gel could be adjusted effectively by changing their stoichiometric ratios.
\end{abstract}

\section{Introduction}

Recently, much effort has been devoted to constructing luminescent materials with efficient high emission in the solid state [1-3]. More and more types of fluorophores with aggregationinduced emission (AIE) characteristics have been discovered and applied in practice [4-6]. Among them, the fluorescent materials emitting circularly polarized luminescence (CPL) have attracted intensive interest owing to their wide applications in various researching fields including 3D displays, chiroptical materials, and so on [7-10]. Circular dichroism (CD) absorption spectra reflect the chirality of the fluorescent materials in the ground state, and circularly polarized luminescence (CPL) spectra reflect the chirality of fluorescent materials in the excited electronic state. Therefore, the CD and CPL spectrum are the two most important tools to test the chirality of luminescent materials $[11,12]$.

As a useful building block in constructing functional materials [13,14], Tröger's base (TB), first synthesized in 1887 [15], shows high controllability and obvious advantages. There are eleven sites in its framework that could be modified without 
considering the side chain. At the same time, the loose stacking of the TB unit and its derivatives, which is caused by its V-configuration could reduce the distance-dependent intermolecular quenching effect in the aggregation state [16]. Moreover, the large dihedral angle of TB $\left(80-104^{\circ}\right)$ [15] permits less selfabsorption and a wider stokes shift [17]. Further, the steric hindrance and highly rigidity could reduce non-radiative transition and restrict the internal rotation [18]. More importantly, in the TB structure, its bridged methylene groups of the diazocine chiral nitrogen atoms prevent the inversion of the configuration, and two stable enantiomers could be formed and separated then Although the TB shows excellent performance in constructing AIE materials, TB-based materials emitting CPL have rarely been reported so far. In general, the luminescent part and the chiral part are necessary to construct CPL-active materials [1921], so the fluorescent Tröger's base derivatives rac-TBPP fall into our sight as the candidate to construct CPL-active materials. Herein, we take two stratgies to construct rac-TBPP-based CPL material. One stratgy is to separate non-CPL emission racTBPP into CPL-active enantiomers $R_{2 N^{-}}$TBPP and $S_{2 N^{-}}$TBPP respectively. The other stratgy is to co-assemble the fluorescent rac-TBPP with the chiral D-glutamic acid gelator DGG to form the CPL-active co-gel. Interestingly, adjusting the stoichiometric ratios of $\mathrm{rac}$-TBPP/DGG of the co-assembling system, the handedness of CPL-active co-gel can be controlled effectively (Scheme 1).

\section{Results and Discussion}

The synthetic routes of rac-TBPP are outlined in Supporting Information File 1, Scheme S1. Firstly, 2,8-dibromo- $6 H, 12 H$ - 5,11-methanodibenzo $[b, f][1,5]$ diazocine was synthesized according to the reported procedure [22]. Then, by Suzuki coupling reaction between 2,8-dibromo- $6 \mathrm{H}, 12 \mathrm{H}$-5,11-methanodibenzo $[b, f][1,5]$ diazocine and 4-vinylpyridine, rac-TBPP was successfully obtained in $51.8 \%$ yield. Detailed experiments and characterization were described in Supporting Information File 1 (Figures S1-S3). Firstly, rac-TBPP was separated into two fractions $R_{2 N^{-}}$TBPP and $S_{2 N^{-}}$TBPP by a chiralpak IB column using MeOH/DCM (80:20, v/v) as the eluent (Supporting Information File 1, Figure S5). The CD spectrum of the first fraction exhibited a positive Cotton effect at $352 \mathrm{~nm}$, assigned to $R_{2 N}$ - TBPP, while the second one showed a negative Cotton effect at the same wavelength, assigned to $S_{2 N^{-}}$TBPP (Figure 1a) [23]. $R_{2 N^{-}}$TBPP and $S_{2 N^{-}}$TBPP were tested further by CPL spectroscopy, and the magnitude of the CPL emission was estimated by a luminescence dissymmetry factor $\left(g_{\text {lum }}\right)$, defined as $2\left(I_{\mathrm{L}}-I_{\mathrm{R}}\right) /\left(I_{\mathrm{L}}+I_{\mathrm{R}}\right)$ where $I_{\mathrm{L}}$ and $I_{\mathrm{R}}$ are the intensity of the left-handed and right-handed CPL signals [24], respectively. Ranging from +2 for an ideal left-handed CPL to -2 for

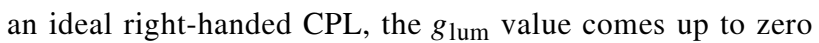
when no circular polarization of the luminescence was detected. The calculated value of $g_{\text {lum }}$ of the CPL signals for $R_{2 N}$-TBPP and $S_{2 N^{-}}$TBPP are +0.0021 , and -0.0025 , repectively (Figure 1b), which is larger than many small organic molecules [25].

In order to avoid a tedious chiral separation of rac-TBPP, we tried to construct the CPL-active material by co-assembling the achiral fluorophore $r a c$-TBPP with a chiral gelator DGG. In the CPL-active co-gels, noncovalent weak interactions might be

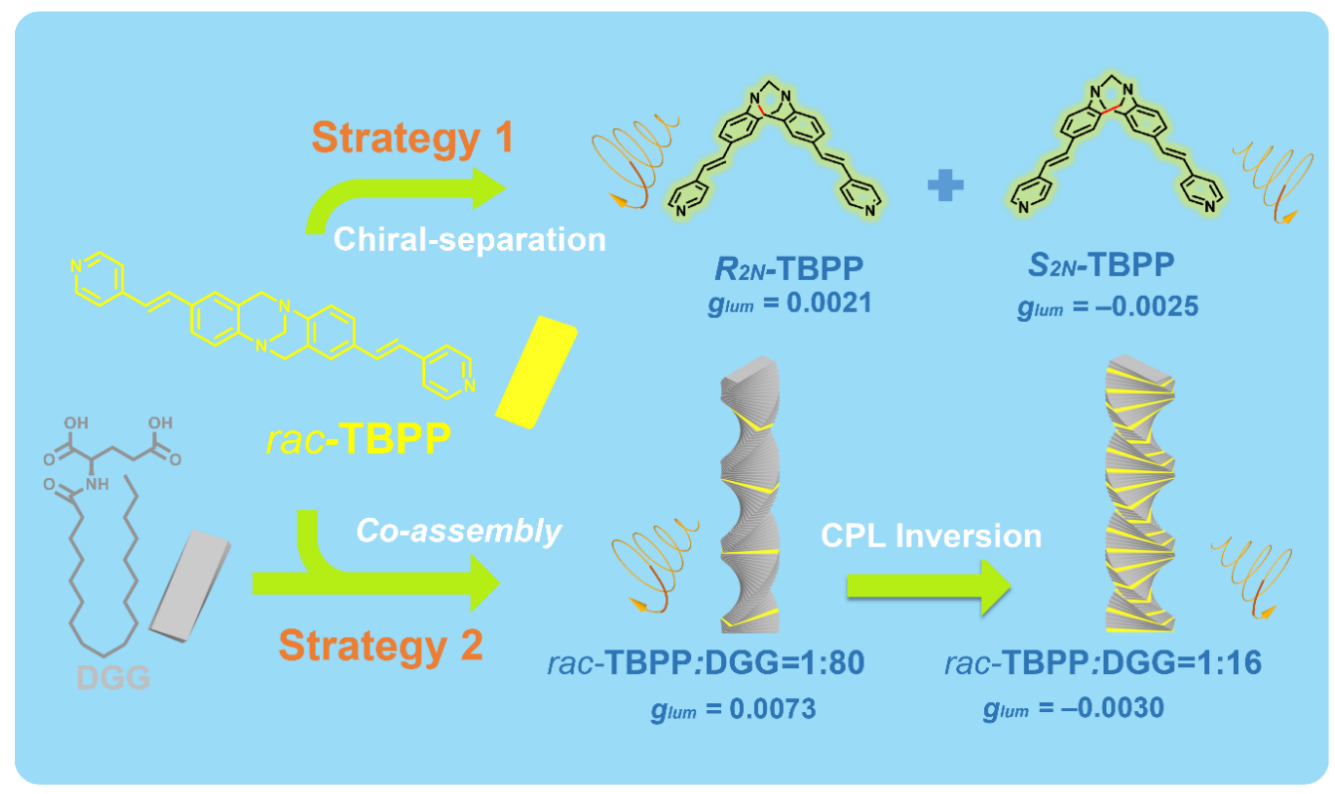

Scheme 1: Cartoon representative for rac-TBPP-based CPL-active systems fabricated through two strategies. 
(a)

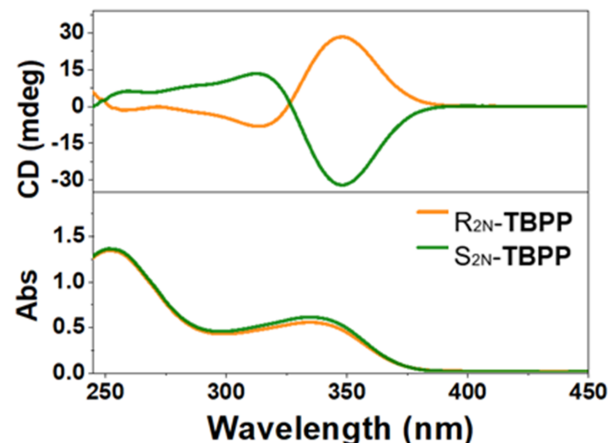

(b)

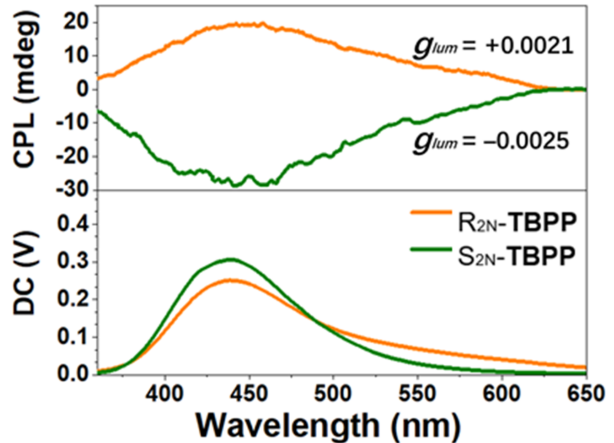

Figure 1: Ground-state and excited-state chirality of $R_{2 N}-$ TBPP and $S_{2 N}-$ TBPP. (a) CD spectra of $R_{2 N^{-}}$TBPP and $S_{2 N}-$ TBPP. (b) CPL spectra of $R_{2 N}$-TBPP and $S_{2 N}$-TBPP excited at $350 \mathrm{~nm}$.

formed between achiral fluorophores and a chiral gelator. So, the CPL emission of co-gels could be adjusted easily by external stimuli. The D-glutamic acid gelator DGG and its enantiomer LGG possess three hydrogen-bond sites, two carboxylic acid groups and one amide, which could be assemblied into the stable spiral structure by hydrogen-bond and other noncovlant interactions. DGG was synthesised by introducing an octadecyl moiety into the glutamic skeleton in $78.6 \%$ yield according to the reported route (Supporting Information File 1, Scheme S2)
[26]. When rac-TBPP was mixed with DGG at molar ratios from 1:100 to 1:16 (rac-TBPP/DGG), transparent yellow co-gels were successfully formed by being heated to dissolve in chloroform, and then cooled to ambient temperature (Supporting Information File 1, Figure S6). Owing to the AIE effect of the TB unit the fluorescence intensity of these co-assembly co-gels enhanced sharply (Figure 2a). More interestingly, at the molar ratio of $\mathrm{rac}$-TBPP/DGG $=1: 16$, the CPL spectra of the co-gel shows a negative signal, while at the molar ratio of rac-

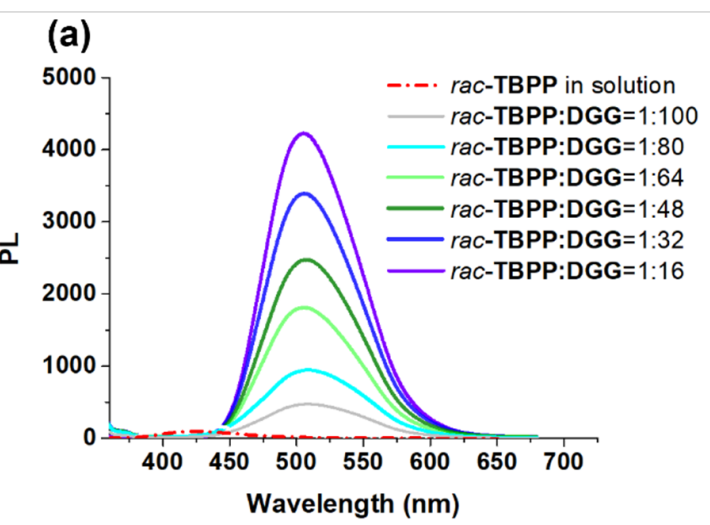

(c)

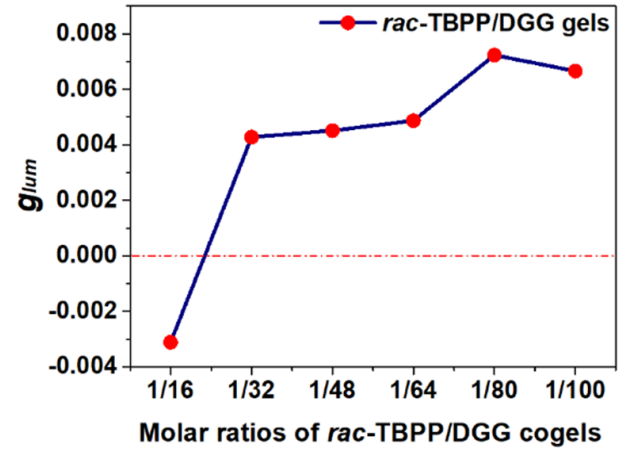

(b)

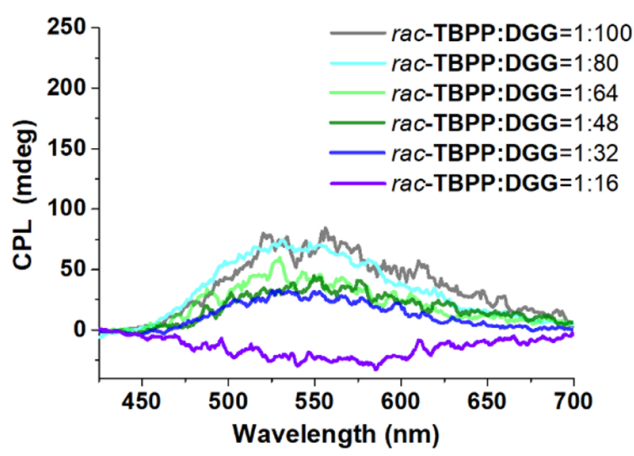

(d)

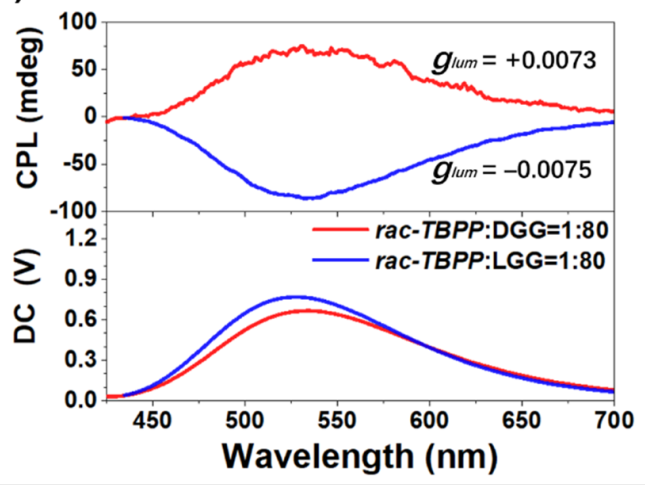

Figure 2: (a) Fluorescence spectra of rac-TBPP in solution (dash line) and in the rac-TBPP/DGG co-gels. (b) CPL spectra of the rac-TBPP/DGG co-gel at molar ratios from 1:100 to 1:16. (c) Plot of $g_{\text {lum }}$ value of CPL signals versus ratios of rac-TBPP/DGG in the co-gel. (d) Mirror symmetry in CPL spectra of rac-TBPP/DGG co-gel (red line) and rac-TBPP/LGG (blue line) co-gel at the molar ratio of 1:80. 
TBPP/DGG $=1: 32$ or higher, positive signals exhibiting lefthanded CPL signals were observed (Figure 2b). At the molar ratio of $\mathrm{rac}$-TBPP/DGG $=1: 80$, the CPL spectra shows a positive signal with a $g_{\text {lum }}$ value about +0.0073 , which was almost three times higher than the $g_{\text {lum }}$ value of TBPP enantiomers (Figure 2c). Compared with the co-gel at the molar ratio of racTBPP/DGG $=1: 80$, a mirror symmetry was observed in the CPL spectra of the co-gel at the molar ratio of $r a c-\mathbf{T B P P} /$ LGG = 1:80 (Figure 2d).

rac-TBPP contains pyridine units, and the gelator DGG has the carboxyl groups. Therefore, hydrogen bonds might be formed between pyridine in rac-TBPP and the carboxyl groups in DGG. Moreover, the morphologies of $\mathrm{rac}$-TBPP/DGG co-gels might be different due to the changing stoichiometric ratios. In order to get an indepth understanding on the inversion of CPL handedness, $r a c$-TBPP/DGG co-gels at molar ratios of 1:16 and 1:80 were explored further by UV-vis and FTIR (fourier transform infrared) spectra. UV-vis absorption spectra of racTBPP/DGG co-gels exhibited a strong absorption band at $333 \mathrm{~nm}$, assigned to the conjugated structure of benzene and pyridine in the rac-TBPP (Figure 3a). However, a red-shift broaden absorption band situated at $372 \mathrm{~nm}$ appears in the racTBPP/DGG co-gel, implying the formation of the ordered packing of rac-TBPP in supramolecular assemblies. At the molar ratio of $\mathrm{rac}$-TBPP/DGG $=1: 80$, The FTIR spectrum was similar to that of the DGG gel, in which $v_{C=O}$ bonds at 1729, 1691 , and $1645 \mathrm{~cm}^{-1}$ reveal that the carboxyl acid groups of DGG could be involved in the formation of various hydrogen bonds (Figure 3b, Figure S7, Supporting Information File 1). At the molar ratio of $\mathrm{rac}$-TBPP/DGG $=1: 16$, the intensity of the peak at $1691 \mathrm{~cm}^{-1}$ decreases, and the peak at $1729 \mathrm{~cm}^{-1}$ brodens. A new peak adjacent to $1645 \mathrm{~cm}^{-1}$ appears at $1627 \mathrm{~cm}^{-1}$. The results demonstrate that some of the acid-acid hydrogen bonds between DGG molecules might be replaced by acid-pyridine hydrogen bonds between DGG and rac-TBPP [27]. In addition, the possible influence of the stoichiometric ratios to the morphologies of $\mathrm{rac}$-TBPP/DGG co-gels was investigated using a scanning electron microscope (SEM). At the molar ratio of $\mathrm{rac}$-TBPP/DGG $=1: 80$, the co-gel shows beltlike nanofibers (Figure 3c), while the fibrous morphology could not be observed at the molar ratio of 1:16 (Figure $3 \mathrm{~d}$ ). It indi- (a)

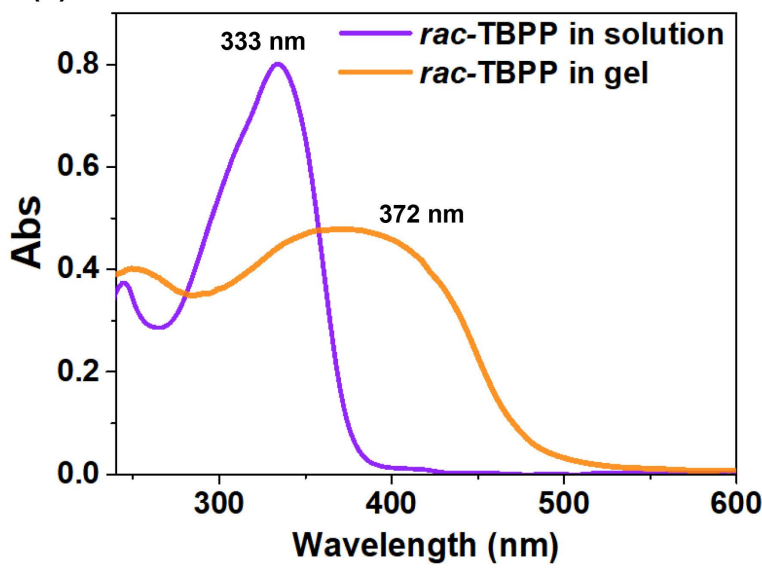

(c)



(b)

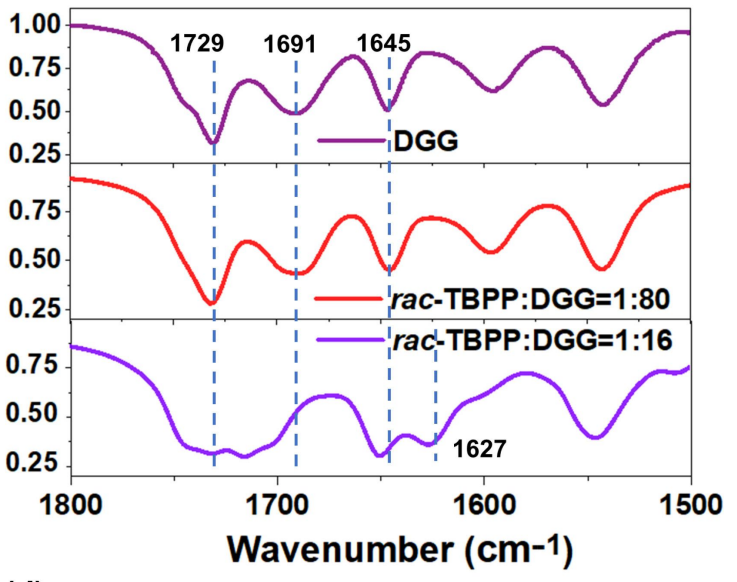

(d)

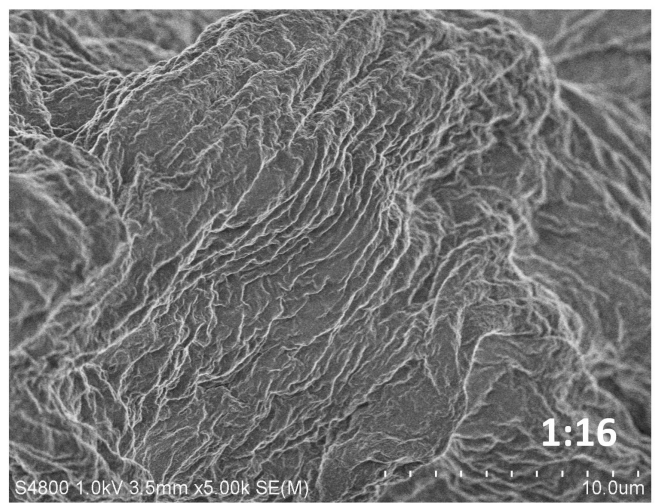

Figure 3: (a) UV-vis absorption spectra of rac-TBPP and rac-TBPP/DGG co-gel (rac-TBPP/DGG = 1:80). (b) FTIR spectra of DGG and rac-TBPP DGG co-gels at molar ratios of 1:80 and 1:16, respectively. SEM images of rac-TBPP/DGG co-gels at molar ratios of 1:80 (c) and 1:16 (d). 
cates that two different kinds of supramolecular assemblies were formed at the ratios of $\mathrm{rac}$-TBPP/DGG $1: 80$ and 1:16, respectively, which is also coincident with the inversion of the CPL responses. However, more detailed studies are still needed to clarify the relations between the morphology and CPL handedness.

\section{Conclusion}

In conclusion, two strategies were demonstrated to obtain CPLactive materials based on the Tröger's base derivative racTBPP. One method is to separate rac-TBPP into two enantiomers $R_{2 N}$-TBPP and $S_{2 N}$-TBPP, which emit strong circularly polarized luminescence. The other strategy is to co-gel the fluorescent $r a c$-TBPP with a chiral D-glutamic acid gelator DGG by the co-assembly strategy. The cogels show significant CPL emission and stoichiometry-controlled inversion of chirality due to the hydrogen bonding interactions and packing modes in the supramolecular co-assemblies. Owing to TB special V-shaped structure, rigid conformation, and nitrogen stereogenic centers make it and its derivatives useful building blocks to construct CPL-active materials and to develop chiral phosphorescent materials in future.

\section{Supporting Information}

\author{
Supporting Information File 1 \\ Experimental part. \\ [https://www.beilstein-journals.org/bjoc/content/ \\ supplementary/1860-5397-17-6-S1.pdf]
}

\section{Acknowledgements}

We thank Prof. Carsten Schmuck for valuable suggestions and discussion on our researchs on molecular recognition during his visit in Nanjing Univ. in 2011 and afterwards.

\section{Funding}

This work was supported by the National Natural Science Foundation of China (Nos. 21901113, 21871135, 21871136), and the Natural Science Foundation of Jiangsu Province (No. BK20190287).

\section{ORCID ${ }^{\circledR}$ iDs}

Juli Jiang - https://orcid.org/0000-0001-5778-6380

Leyong Wang - https://orcid.org/0000-0001-5775-3714

\section{Preprint}

A non-peer-reviewed version of this article has been previously published as a preprint: https://doi.org/10.3762/bxiv.2020.112.v1

\section{References}

1. Zhang, Z.-Y.; Chen, Y.; Liu, Y. Angew. Chem., Int. Ed. 2019, 58, 6028-6032. doi:10.1002/anie.201901882

2. Chen, C.; Chi, Z.; Chong, K. C.; Batsanov, A. S.; Yang, Z.; Mao, Z.; Yang, Z.; Liu, B. Nat. Mater. 2020. doi:10.1038/s41563-020-0797-2

3. Bolton, O.; Lee, K.; Kim, H.-J.; Lin, K. Y.; Kim, J. Nat. Chem. 2011, 3, 205-210. doi:10.1038/nchem.984

4. Zhao, Z.; Zhang, H.; Lam, J. W. Y.; Tang, B. Z. Angew. Chem., Int. Ed. 2020, 59, 9888-9907. doi:10.1002/anie.201916729

5. Li, J.; Wang, J.; Li, H.; Song, N.; Wang, D.; Tang, B. Z. Chem. Soc. Rev. 2020, 49, 1144-1172. doi:10.1039/c9cs00495e

6. Roose, J.; Tang, B. Z.; Wong, K. S. Small 2016, 12, 6495-6512. doi:10.1002/smll.201601455

7. Han, J.; Guo, S.; Lu, H.; Liu, S.; Zhao, Q.; Huang, W. Adv. Opt. Mater. 2018, 6, 1800538. doi:10.1002/adom.201800538

8. Zheng, H.; Li, W.; Li, W.; Wang, X.; Tang, Z.; Zhang, S. X.-A.; Xu, Y. Adv. Mater. (Weinheim, Ger.) 2018, 30, 1705948. doi:10.1002/adma.201705948

9. Nitti, A.; Pasini, D. Adv. Mater. (Weinheim, Ger.) 2020, 32, 1908021. doi:10.1002/adma.201908021

10. Han, J.; You, J.; Li, X.; Duan, P.; Liu, M. Adv. Mater. (Weinheim, Ger.) 2017, 29, 1606503. doi:10.1002/adma.201606503

11. Berova, N.; Bari, L. D.; Pescitelli, G. Chem. Soc. Rev. 2007, 36, 914-931. doi:10.1039/b515476f

12. Sánchez-Carnerero, E. M.; Agarrabeitia, A. R.; Moreno, F.; Maroto, B. L.; Muller, G.; Ortiz, M. J.; de la Moya, S. Chem. - Eur. J. 2015, 21, 13488-13500. doi:10.1002/chem.201501178

13. Rúnarsson, Ö. V.; Artacho, J.; Wärnmark, K. Eur. J. Org. Chem. 2012, 7015-7041. doi:10.1002/ejoc.201201249

14. Dolenský, B.; Havlík, M.; Král, V. Chem. Soc. Rev. 2012, 41 3839-3858. doi:10.1039/c2cs15307f

15. Tröger, J. J. Prakt. Chem. 1887, 36, 225-245. doi:10.1002/prac.18870360123

16. Yuan, C.-X.; Tao, X.-T.; Ren, Y.; Li, Y.; Yang, J.-X.; Yu, W.-T.; Wang, L.; Jiang, M.-H. J. Phys. Chem. C 2007, 111, 12811-12816. doi:10.1021/jp0711601

17. Xi, H.; Liu, Y.; Yuan, C.-X.; Li, Y.-X.; Wang, L.; Tao, X.-T.; Ma, X.-H.; Zhang, C.-F.; Hao, Y. RSC Adv. 2015, 5, 45668-45678. doi:10.1039/c5ra07912h

18. Yuan, R.; Li, M.-q.; Xu, J.-b.; Huang, S.-y.; Zhou, S.-I.; Zhang, P.; Liu, J.-j.; Wu, H. Tetrahedron 2016, 72, 4081-4084. doi:10.1016/j.tet.2016.05.042

19. Sang, Y.; Han, J.; Zhao, T.; Duan, P.; Liu, M. Adv. Mater. (Weinheim, Ger.) 2020, 32, 1900110. doi:10.1002/adma.201900110

20. Takaishi, K.; Iwachido, K.; Takehana, R.; Uchiyama, M.; Ema, T. J. Am. Chem. Soc. 2019, 141, 6185-6190. doi:10.1021/jacs.9b02582

21. Liang, J.; Guo, P.; Qin, X.; Gao, X.; Ma, K.; Zhu, X.; Jin, X.; Xu, W.; Jiang, L.; Duan, P. ACS Nano 2020, 14, 3190-3198. doi:10.1021/acsnano.9b08408

22. Yuan, C.; Zhang, Y.; Xi, H.; Tao, X. RSC Adv. 2017, 7, 55577-55581. doi:10.1039/c7ra11228a

23. Chen, Y.; Cheng, M.; Hong, B.; Zhao, Q.; Qian, C.; Jiang, J.; Li, S.; Lin, C.; Wang, L. Front. Chem. (Lausanne, Switz.) 2019, 7, 383. doi:10.3389/fchem.2019.00383

24. Riehl, J. P.; Richardson, F. S. Chem. Rev. 1986, 86, 1-16. doi:10.1021/cr00071a001

25. Ma, J.-L.; Peng, Q.; Zhao, C.-H. Chem. - Eur. J. 2019, 25 , 15441-15454. doi:10.1002/chem.201903252 
26. Bachl, J.; Mayr, J.; Sayago, F. J.; Cativiela, C.; Díaz Díaz, D.

Chem. Commun. 2015, 51, 5294-5297. doi:10.1039/c4cc08593k

27. Li, P.; Lü, B.; Han, D.; Duan, P.; Liu, M.; Yin, M. Chem. Commun.

2019, 55, 2194-2197. doi:10.1039/c8cc08924h

\section{License and Terms}

This is an Open Access article under the terms of the Creative Commons Attribution License

(https://creativecommons.org/licenses/by/4.0). Please note that the reuse, redistribution and reproduction in particular requires that the author(s) and source are credited and that individual graphics may be subject to special legal provisions.

The license is subject to the Beilstein Journal of Organic Chemistry terms and conditions:

(https://www.beilstein-journals.org/bjoc/terms)

The definitive version of this article is the electronic one which can be found at:

https://doi.org/10.3762/bjoc.17.6 